\title{
Optical conductivity and the correlation strength of high-temperature copper-oxide superconductors
}

\author{
ARMIN COMANAC'1 , LUCA DE' MEDICl2, MASSIMO CAPONE 3 ,4 AND A. J. MILLIS ${ }^{1 *}$ \\ ${ }^{1}$ Department of Physics, Columbia University, 538 W. 120th Street, New York, New York 10027, USA \\ ${ }^{2}$ Department of Physics and Center for Materials Theory, Rutgers the State University of NJ, 136 Frelinghuysen Road, Piscataway, New Jersey 08854, USA \\ ${ }^{3}$ SMC, CNR-INFM, and Dipartimento di Fisica, Universit di Roma 'La Sapienza', Piazzale A. Moro 2, I-00185, Rome, Italy \\ ${ }^{4}$ ISC-CNR, Via dei Taurini 18, I-00185, Rome, Italy \\ *e-mail: millis@phys.columbia.edu
}

Since their discovery in 1986, the high-temperature copper-oxide superconductors have been a central object of study in condensed-matter physics. Their highly unusual properties are widely (although not universally) believed to be a consequence of electron-electron interactions that are so strong that the traditional paradigms of condensed-matter physics do not apply: instead, entirely new concepts and techniques are required to describe the physics. In particular, the superconductivity is obtained by adding carriers to insulating 'parent compounds'. These parent compounds have been identified' as 'Mott' insulators, in which the lack of conduction arises from anomalously strong electron-electron repulsion. The unusual properties of Mott insulators are widely ${ }^{2}$ believed to be responsible for the high-temperature superconductivity. Here, we present a comparison of new theoretical calculations and published ${ }^{3-8}$ optical conductivity measurements, which challenges this belief. The analysis indicates that the correlation strength in the cuprates is not as strong as previously believed, in particular that the materials are not properly regarded as Mott insulators. Rather, antiferromagnetism seems to be necessary to obtain the insulating state. By implication, antiferromagnetism is essential to the properties of the doped metallic and superconducting state as well.

The prototypical 'parent compound' is $\mathrm{La}_{2} \mathrm{CuO}_{4}$, in which the lattice structure and electron counting is such that there is an odd number of electrons per formula unit. Thus, in the absence of further symmetry breaking, conventional band theory would predict that the material is a good metal. $\mathrm{La}_{2} \mathrm{CuO}_{4}$ is however not metallic; it is an insulator with a gap determined by optical spectroscopy to be approximately $1.8 \mathrm{eV}$ (refs 3,4). From one perspective, the insulating behaviour is not surprising. At temperature $T=0, \mathrm{La}_{2} \mathrm{CuO}_{4}$ has two-sublattice Néel order, so that the magnetic unit cell contains two formula units and thus an even number of electrons, compatible with the observed insulating behaviour. However, the consensus has been that the antiferromagnetic order is irrelevant. Instead, the materials have been identified ${ }^{1,2}$ as 'Mott insulators'. (Although the cuprates are properly regarded as 'charge-transfer' and not 'Mott' insulators in the sense of ref. 9, we believe this issue is not relevant here: the high-energy-scale physics and chemistry of transition metal
$(\mathrm{Cu})$ and ligand $(\mathrm{O})$ ions produces one band of electrons, with an effective interaction strength $U$ which we aim to determine. In particular, optical data show that the nearest bands (arising mainly from the non-bonding oxygen orbitals) are 5-6 eV removed in energy, with only a weak absorption tail extending down to the energies of relevance here. The issue is discussed in more detail in the Supplementary Information.) In a Mott or charge-transfer insulator, the electron-electron interactions are so strong that a density of one electron per unit cell implies a 'jammed' situation: no electron can move without creating an energetically expensive doubly occupied site. Removing or adding electrons creates 'holes' or doubly occupied sites, whose motion is not blocked by the jamming effect but is strongly affected by the non-trivial Mott insulating background in which it moves ${ }^{2}$.

Important experimental evidence bearing on the question of the nature of the insulating state comes from the optical (frequency-dependent) conductivity, $\sigma(\omega)$ : the linear response function connecting a frequency-dependent, transverse electric field $\mathbf{E}$ to the current $\mathbf{j}$ it induces. At frequencies less than the interband threshold, the measured conductivity is dominated by processes in which an electron moves from one unit cell to another. In a Mott insulator, such conductivity processes are suppressed by the blocking effect of onsite repulsion ${ }^{10}$, so that the expected lowfrequency spectral weight (integrated optical absorbtion strength) is small. Here, we show that in the high- $T_{\mathrm{c}}$ materials the measured low-energy spectral weight is too large to be compatible with the Mott (blocking) interpretation of the physics of the cuprates.

The electronic structure of the cuprates is such that one band (per $\mathrm{CuO}_{2}$ unit) crosses the chemical potential; all other bands are full or empty and may to first approximation be neglected. Electrons moving in the relevant band are subject to an interaction whose most important component is a repulsion disfavouring configurations in which two electrons occupy the same site at the same time. This physics may be expressed mathematically through the 'Hubbard' model of a band of electrons subject to local correlations. Although the Hubbard model is not a fully accurate description of the physics of high-temperature superconductors, it contains the essence of the blocking effect and is generally accepted ${ }^{2}$ as the basic picture on which a more refined description should be based. (Preliminary studies of charge-transfer models presented 
a

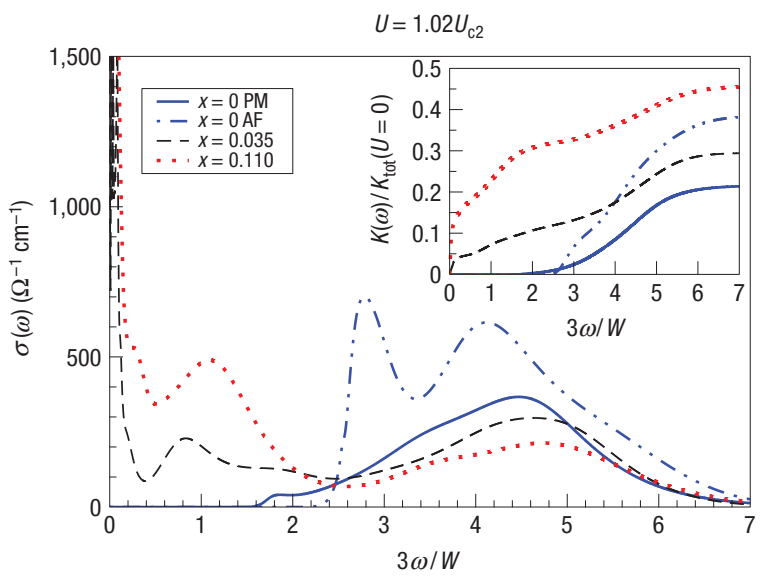

b

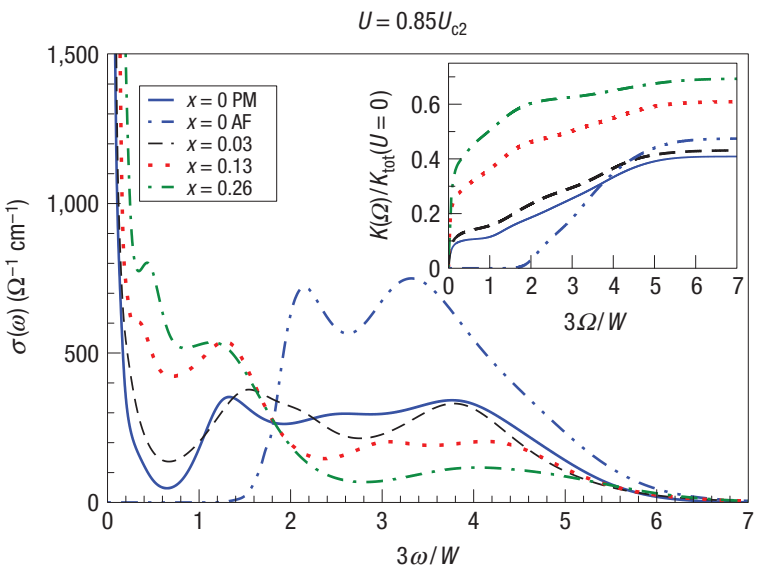

Figure 1 Optical conductivity of Hubbard model. Calculated as described in the main text at dopings $x=1-n$ and interaction strength $U$ indicated. a,b, Optical conductivity. Insets: Optical integral. For $x=0$, both paramagnetic (PM) and antiferromagnetic (AF) phase calculations are shown; for $x>0$, only paramagnetic phase results are given. If the band theory value $W=3 \mathrm{eV}$ is used then the frequency scale is electronvolts.

in the Supplementary Information are in agreement with this conclusion.) We write the model in a mixed momentum $(k)$ space position (i) space representation as

$$
H=\sum_{k, \sigma} \varepsilon_{k} c_{k, \sigma}^{\dagger} c_{k \sigma}+U \sum_{i} \hat{n}_{i, \uparrow} \hat{n}_{i, \downarrow}
$$

Here, $c_{k \sigma}^{\dagger} c_{k \sigma}$ creates (annihilates) an electron of spin $\sigma$ in momentum state $k, U$ is the effective 'blocking' interaction, $\hat{n}_{i, \sigma}$ is the density operator for electrons of spin $\sigma$ on site $i$ and $\varepsilon_{k}$ is the dispersion given by local density band calculations. Small variations among different calculations exist, but all agree within a few per cent on the values of the parameters important for this study, which are the bandwidth $W \approx 3 \mathrm{eV}$ and the 'kinetic energy' $K \approx 0.4 \mathrm{eV}$. (For definiteness, we use the $\varepsilon_{k}$ derived from the 'downfolding' parametrization of ref. 11.)

At a density of one electron per cell the ground state of $H$, equation (1), is believed to be a paramagnetic metal at small $U$ (roughly $U<1 \mathrm{eV}$ ) and an antiferromagnetic insulator at larger $U$, with a small range of antiferromagnetic metal in between. The key question is whether the antiferromagnetic order is essential to the insulating nature of the ground state. To determine this we turn to the single-site dynamical mean-field approximation ${ }^{12}$. In this approximation, spatial correlations between fluctuations are neglected but temporal fluctuations on a given site are included exactly. If long-ranged antiferromagnetic order is not included in the calculation, we find at carrier concentration $n=1$ and temperature $T=0$ a critical value $U_{\mathrm{c} 2} \approx 1.45 \mathrm{~W}$ separating a small $U$ metallic phase from a large $U$ insulating phase. The band theory estimate $W \approx 3 \mathrm{eV}$ implies $U_{\mathrm{c} 2} \approx 4.4 \mathrm{eV}$. This large $U$ phase is identified as a Mott insulator because an energy gap exists at the chemical potential in the absence of any intersite magnetic correlations.

We calculated the optical conductivity implied by equation (1), representing the electric field by a vector potential $\mathbf{A}$, using the minimal coupling $k \rightarrow k-A$ and standard linear response theory and multiplying the calculated result (a dimensionless conductance per $\mathrm{CuO}_{2}$ plane) by the conductance quantum $e^{2} / \hbar$ and dividing by a typical interplane distance $d=6 \AA$. Figure la,b shows the calculated conductivity at several carrier densities for a $U$ slightly greater than $U_{\mathrm{c} 2}$ and a $U$ slightly less than $U_{\mathrm{c} 2}$. Consider the $x=0$ results, representative of the parent compounds of the high- $T_{\mathrm{c}}$ materials. The $U>U_{\mathrm{c} 2}$ calculation reveals Mott insulating behaviour: even if magnetic order is neglected, the result is insulating (gap in the conductivity spectrum). Adding antiferromagnetism increases the gap and produces structure at the gap edge. On the other hand, the $U<U_{\mathrm{c} 2}$ calculation reveals metallic behaviour (no gap) in the absence of antiferromagnetism, whereas the antiferromagnetic calculation reveals a large gap.

To interpret the results we note that in models such as equation (1) the optical conductivity obeys a 'restricted f-sum rule ${ }^{10,13}$. Defining

$$
K(\Omega)=\left(\frac{V_{\text {cell }}}{a^{2}}\right) \int_{0}^{\Omega} \frac{2 \mathrm{~d} \omega}{\pi} \frac{\sigma(\omega)}{\sigma_{\mathrm{Q}}},
$$

we have

$$
K(\infty)=\sum_{k, \sigma} n_{k, \sigma} \frac{\partial^{2} \varepsilon_{k}}{\partial k_{x}^{2}} .
$$

Here $\sigma_{\mathrm{Q}}=e^{2} / \hbar$ is the conductance quantum, $V_{\text {cell }}$ is the volume of the unit cell, $a$ is the in-plane lattice constant and $n_{k, \sigma}$ is the probability that the state of momentum $k$ and spin $\sigma$ is occupied. Note that $\sigma(\omega)$ in equation (2) refers to the real (dissipative) conductivity calculated from equation (1); in physical terms, it corresponds to that contribution to the measured conductivity arising from transitions within the band of states described by equation (1). In a real material, interband transitions not described by equation (1) also contribute to the conductivity; these make up the difference between equation (3) and the familiar f-sum rule $\int_{0}^{\infty} \mathrm{d} \omega \sigma(\omega)=\pi n e^{2} / 2 m$.

If $U=0, n_{k, \sigma}$ is the usual Fermi-Dirac distribution, corresponding to filling up only the lowest-lying states in the band. Evaluation of equation (3) for this case yields $K=K_{\text {band }} \approx 0.4 \mathrm{eV}$, essentially independent of carrier concentration for the dopings relevant to high-temperature superconductivity. In this non-interacting case, the electrons are not scattered: they are freely accelerated by an applied electric field so the conductivity is just a delta function of strength $\pi K$ at $\omega=0$. Increasing the interaction causes electron-electron scattering which shifts the spectral weight from $\omega=0$ to higher frequencies. Increasing the interaction also tends to localize the electrons, leading to an $n(k)$ more uniformly distributed over the band and thus reducing the magnitude of the integral in equation (3), that is, decreasing the 


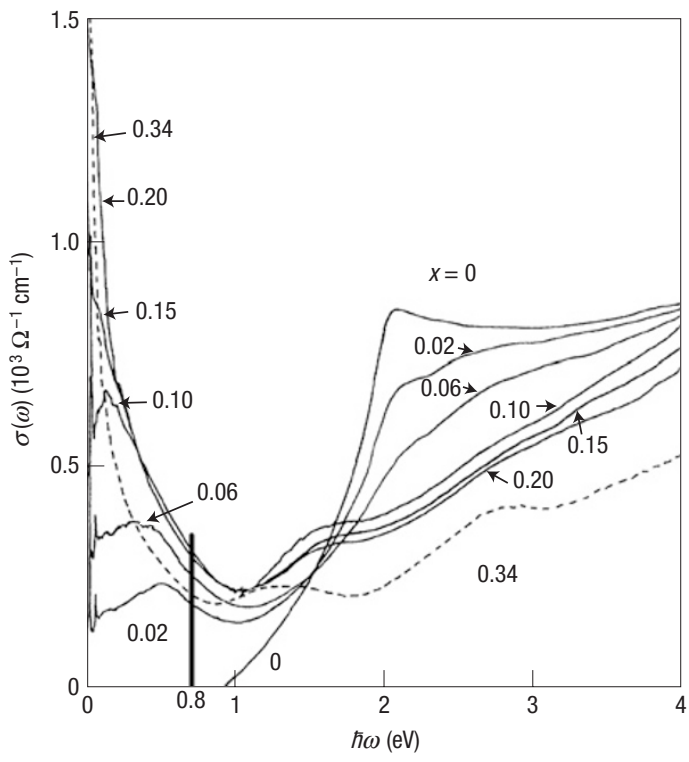

Figure 2 Measured optical conductivity of $\mathrm{La}_{2-x} \mathrm{Cu}_{x} \mathrm{CuO}_{4}$ reproduced from ref. 4. The solid vertical line at frequency $\omega=0.8 \mathrm{eV}$ indicates cutoff frequencies used for spectral weight analysis.

total spectral weight. However, adding holes allows carrier motion, thus increasing the spectral weight and shifting it back towards $\omega=0$. These effects can be seen in the insets of Fig. 1, which plot the conductivity integral, equation (2) obtained from the calculated conductivities shown in the main panels of the figure.

We now compare the calculation to measurements of the conductivity, of which a representative example ${ }^{4}$ is shown in Fig. 2. These data were taken in 1991; subsequent improvements especially in sample quality have sharpened the bandgap seen in the conductivity of the $x=0$ sample, so that the onset of absorbtion begins at $\omega \approx 1.8 \mathrm{eV}$, but have not changed the material features, in particular the spectral weights in the different frequency regimes. Use of the band theory estimate $W \approx 3 \mathrm{eV}$ would imply the bandgap is approximately $0.6 \mathrm{~W}$, consistent with the result of the antiferromagnetic-phase $U<U_{\mathrm{c} 2}$ calculation but inconsistent with the antiferromagnetic-phase $U>U_{\mathrm{c} 2}$ result. The antiferromagnetic $U>U_{\mathrm{c} 2}$ calculation can be made consistent with the observed bandgap by reducing the energy scales by $25 \%$, implying in particular a bandwidth $W^{*} \approx 2.25 \mathrm{eV}$ instead of the $W \approx 3 \mathrm{eV}$ found in band theory calculations. However, even if this renormalization is made, the magnitude of the observed conductivity is inconsistent with the $U>U_{\mathrm{c} 2}$ hypothesis, as will now be shown.

The measured spectral weight in the range $\omega<3 \mathrm{eV}$ for the insulating compound corresponds to $K(3 \mathrm{eV})=0.2 \mathrm{eV}$ or about $50 \%$ of the non-interacting value. It is likely that not all of the spectral weight observed in the range below $3 \mathrm{eV}$ is due to the optical transitions of interest. Interband transitions to irrelevant bands may contribute. To obtain an upper bound on possible interband contributions, we note that as doping increases, the calculated conductivity shifts strongly to lower frequencies (as may be seen in Fig. 1). We therefore use the measured $x=0.34$ data in the range $\omega>1 \mathrm{eV}$ as an estimate of the interband contribution to the conductivity. We have integrated the difference between the conductivity measured in the $x=0$ sample and that measured in the $x=0.34$ sample over the range $\omega<3 \mathrm{eV}$, obtaining $K_{\exp }(\Omega=3 \mathrm{eV}) \approx 0.2 K_{\text {band }} \approx 0.1 \mathrm{eV}$. This estimate is

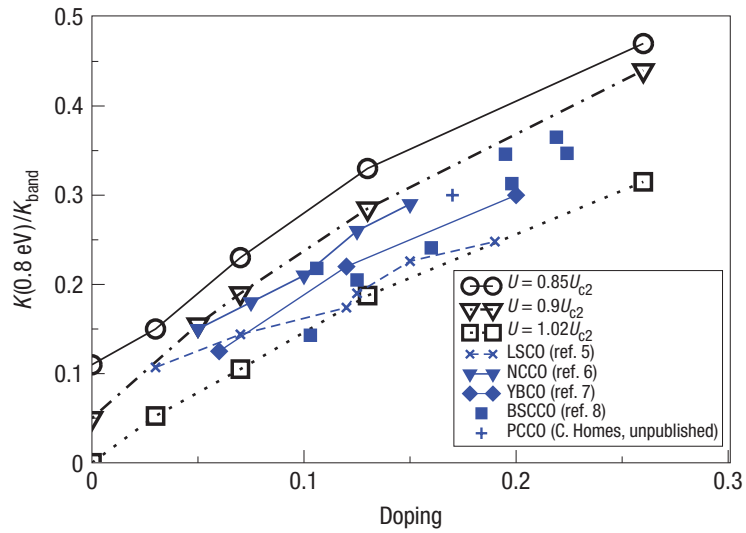

Figure 3 Comparison of measured and calculated optical spectral weight. Filled symbols: spectral weight obtained by integrating experimental conductivity up to $0.8 \mathrm{eV}$ from references given. Open symbols: theoretically calculated spectral weight, integrated up to $W / 4$. For $U=0.85 U_{\mathrm{c} 2}$ and $U=0.9 U_{\mathrm{c} 2}$, the band-theory estimate $W=3 \mathrm{eV}$ is used to convert the calculation to physical units; for $U=1.02 U_{\mathrm{c} 2}$, the value $W=2.25 \mathrm{eV}$ which reproduces the insulating gap is used.

quite consistent with the results shown in Fig. 1b, inset. However, the $U>U_{\mathrm{c} 2}$ calculation yields substantially less spectral weight in the low-frequency regime. Combining the band theory estimate $W \approx 3 \mathrm{eV}$ with the data in Fig. 1a, inset yields $K(3 \mathrm{eV}) \approx 0.03 \mathrm{eV}$, far less than the measured $0.1 \mathrm{eV}$. If we use instead the renormalized $W^{*}=2.25 \mathrm{eV}$ which reproduces the value of the gap, then $3 \mathrm{eV} \approx 4 W^{*} / 3$. The total spectral weight integrated up to this point is $0.13 K(U=0)$ and because the theoretical $K(U=0) \sim W$ this implies an integrated weight of about $0.05 \mathrm{eV}$, still much smaller than what is observed.

Now $\mathrm{La}_{2} \mathrm{CuO}_{4}$ is observed to remain insulating at temperatures above its Néel temperature $\approx 340 \mathrm{~K}$, so long-ranged order is not essential to the insulating behaviour. However, the Nél temperature is strongly suppressed by low-dimensional fluctuation effects and is a poor measure of the strength of the magnetic correlations, which are found to remain significant up to the highest measured temperatures ${ }^{14}(T \approx 1,000 \mathrm{~K})$. Recent cluster dynamical mean-field calculations ( $\mathrm{K}$. Haule et al., private communication; E. Gull, P. Werner, M. Troyer and A. Millis, manuscript in preparation) produce insulating behaviour over wide temperature ranges without long-ranged order, even in the intermediate coupling regime, provided that near-neighbour spin correlations are strong enough.

Next, we turn to the doping dependence of the conductivity. The filled symbols in Fig. 3 show the optical spectral weight for several cuprate materials, integrated up to $0.8 \mathrm{eV}$, about 0.45 of the insulating gap. The value is chosen because available evidence indicates that the conductivity at $\omega<1 \mathrm{eV}$ is essentially uncontaminated by interband transitions, whereas at higher frequencies, the situation is less clear ${ }^{15}$. It can be seen that the measured spectral weight in the midgap region scales linearly with doping, but with a non-vanishing intercept. The open symbols show the results of the theoretical calculations for a $U$ slightly greater than $U_{\mathrm{c} 2}$ and for two $U$ values less than $U_{\mathrm{c} 2}$. For the $U>U_{c 2}$ calculation, we have used the scale $W^{*}=2.25 \mathrm{eV}$ to convert the theoretical results to physical units. We see that for $U>U_{\mathrm{c} 2}$ the calculated spectral weight is qualitatively inconsistent with the data ${ }^{16}$, because it vanishes as doping tends to zero. However, we note that in the qualitative comparison, the decisive feature is the behaviour at $x<0.1$ where the uncertainties in 
the data are largest. Further experimental examination of this frequency regime would be desirable. The $U>U_{\mathrm{c} 2}$ results are also smaller in magnitude than the experimentally determined values. On the other hand, the results for $U=0.9 U_{\mathrm{c} 2}$ give a magnitude and doping dependence that is reasonably consistent with the measured values at non-vanishing dopings. The $x>0$ calculations are carried out within single-site dynamical meanfield theory in the paramagnetic phase. This method does not take into account the effects of near-neighbour magnetic correlations, which are likely to be present even in the absence of true longranged order and which will suppress the spectral weight in the low-frequency regime. We suggest that a model with a $U \approx 0.85 U_{\mathrm{c} 2}$ and with a proper treatment of antiferromagnetic correlations will lead to a doping dependence of the spectral weight that is consistent with experiment.

This paper draws conclusions on the basis of the comparison of a one-band model to data. At sufficiently high energies, other bands and other states in the $d$ multiplet will become important. Although the Supplementary Information presents evidence that the effects of other bands appear only at energies higher than those relevant for our conclusions, the issue clearly warrants a more thorough examination, and our conclusions should be regarded as tentative until this examination is carried out. Our findings suggest that a re-examination of theoretical approaches to high-temperature superconductivity would be worthwhile. Much work has been based on the ' $t-J$ ' model $^{2}$, which is derived on the assumption that the correlation-induced blocking effect is fundamental, with antiferromagnetism providing a next correction and which has been widely accepted because it provides a natural explanation of the striking doping dependence of physical properties. Determining whether the observed doping dependence of the low-energy physics can be understood within the intermediate-coupling, strong antiferromagnetic correlations picture implied by the optical data, is an important challenge for future work.

\section{METHODS}

Theoretical results were obtained using the single-site dynamical mean-field method. The equations were solved by two methods: continuous-time quantum Monte Carlo ${ }^{17}$ (CT-QMC) plus maximum entropy analytical continuation ${ }^{18}$ and $T=0$ exact diagonalization ${ }^{19,20}$. The CT-QMC results presented here are all obtained at the very low temperature $T=W / 800$ except for the two antiferromagnetic cases, where higher temperatures $T=W / 40\left(U=0.85 U_{\mathrm{c} 2}\right)$ and $T=W / 56\left(U=1.02 U_{\mathrm{c} 2}\right)$ were needed to stabilize the analytical continuation. In all cases, the temperatures were low compared with the relevant scales in the problem; the results may be taken as representative of $T=0$ except for a slight broadening of the above-gap peak in the $U=0.85 U_{\mathrm{c} 2}$ antiferromagnetic results. The two methods, which have independent sources of error, produce results which agreed at the $5 \%$ level. Representative results are shown in Supplementary Information, Fig. S1, which presents conductivities calculated by the two methods, and integrals over various frequency regimes.

The calculational results shown used a semicircular density of states with bandwidth adjusted to match the tight-binding band theory values.
Unfortunately, the $T=0$ exact diagonalization method has not yet produced a stable solution for the tight-binding density of states, and although the CT-QMC method has produced stable imaginary-time solutions, we have not succeeded in obtaining a reasonable analytical continuation for the tight-binding density of states. Imaginary-time CT-QMC calculations were used to verify that the critical value for the Mott transition and the total kinetic energy obtained for the tight-binding density of states agreed to within a few per cent of the values obtained for the semicircular one if the bandwidths were matched.

Received 29 August 2007; accepted 14 January 2008; published 17 February 2008

\section{References}

1. Anderson, P. W. The resonating valence bond state in $\mathrm{La}_{2} \mathrm{CuO}_{4}$ and superconductivity. Science 235 1196-1198 (1987)

2. Lee, P. A., Nagaosa, N. \& Wen, X.-G. Doping a Mott insulator: Physics of high-temperature superconductivity. Rev. Mod. Phys. 78, 17-86 (2006).

3. Basov, D. \& Timusk, T. Electrodynamics of high- $T_{\mathrm{c}}$ superconductors. Rev. Mod. Phys. 77, 721-780 (2005).

4. Uchida, S. et al. Optical spectra of $\mathrm{La}_{2-x} \mathrm{Sr}_{x} \mathrm{CuO}_{4}$ : Effect of carrier doping on the electronic structure of the $\mathrm{CuO}_{2}$ plane. Phys. Rev. B 43, 7942 (1991).

5. Lucarelli, A. et al. Phase diagram of $\mathrm{La}_{2-x} \mathrm{Sr}_{x} \mathrm{CuO}_{4}$ probed in the infrared: Imprints of charge stripe excitations. Phys. Rev. Lett. 90, 037002 (2003).

6. Onose, Y., Taguchi, Y., Ishizaka, K. \& Tokura, Y. Charge dynamics in underdoped $\mathrm{Nd}_{2-1} \mathrm{Ce}_{2} \mathrm{CuO}_{4}$ : Pseudogap and related phenomena. Phys. Rev. B 69, 024504 (2004).

7. Cooper, S. L. et al. Optical studies of the a-, b-, and c-axis charge dynamics in $\mathrm{YBa}_{2} \mathrm{Cu}_{3} \mathrm{O}_{6+x}$. Phys. Rev. B 47, 8233-8248 (1993).

8. Hwang, J., Timusk, T. \& Gu, G. D. J. Doping dependent optical properties of $\mathrm{Bi}_{2} \mathrm{Sr}_{2} \mathrm{CaCu}_{2} \mathrm{O}_{8+\delta}$. Phys. Condens. Matter 19, 125208 (2007).

9. Zaanen, J., Sawatzky, G. A. \& Allen, J. Band-gaps and electronic structure of transition-metal compounds. Phys. Rev. Lett. 55, 418-421 (1985).

10. Millis, A. J. in Strong Interactions in Low Dimensions (eds Baeriswyl, D. \& Degiorgi, L.) (Springer, Berlin, 2004).

11. Andersen, O. K., Liechtenstein, A. I., Jepsen, O. \& Paulsen, F. LDA energy bands, low-energy Hamiltonians, $\mathrm{t}^{\prime}, \mathrm{t}^{\prime \prime}, \mathrm{t}$ perpendicular to (k), and J perpendicular to (k). J. Phys. Chem. Solids 56, 1573-1591 (1995)

12. Georges, A., Kotliar, B. G., Krauth, W. \& Rozenberg, M. J. Dynamical mean-field theory of strongly correlated fermion systems and the limit of infinite dimensions. Rev. Mod. Phys. 68, 13-125 (1996).

13. Maldague, P. F. Optical spectrum of a Hubbard chain. Phys. Rev. B 16, 2437 (1977).

14. Imai, T., Slichter, C. P., Yoshimura, K., Katoh, M. \& Kosuge, K. Spin-spin correlation in the quantum critical regime of $\mathrm{La}_{2} \mathrm{CuO}_{4}$. Phys. Rev. Lett. 71, 1254-1257 (1993).

15. Millis, A. J., Zimmers, A., Lobo, R. P. S. M., Bontemps, N. \& Homes, C. C. Mott physics and the optical conductivity of electron-doped cuprates. Phys. Rev. B 72, 24517 (2005).

16. Toschi, A. et al. Temperature dependence of the optical spectral weight in the cuprates: Role of electron correlations. Phys. Rev. Lett. 95, 097002 (2005).

17. Werner, P., Comanac, A., De Medici, L., Troyer, M. \& Millis, A. J. Continuous time solver fo quantum impurity models. Phys. Rev. Lett. 97, 07640 (2006).

18. Gubernatis, J. E., Jarrell, M., Silver, R. H. \& Silva, D. S. Quantum Monte Carlo simulations and maximum entropy: Dynamics from imaginary-time data. Phys. Rev. B 44, 6011 (1991).

19. Caffarel, M. \& Krauth, W. Exact diagonalization approach to correlated fermions in infinite dimensions: Mott transition and superconductivity. Phys. Rev. Lett. 72, 1545 (1994).

20. Capone, M., Civelli, M., Kancharla, S. S., Castellani, C. \& Kotliar, G. Cluster-dynamical mean-field theory of the density-driven Mott transition in the one-dimensional Hubbard model. Phys. Rev. B 69 195105 (2004).

\section{Acknowledgements}

This collaboration was begun with support from the Columbia/Polytechnique/Science Po/Sorbonne Alliance program. A.J.M. and A.C. were supported by NSF-DMR-0705847 and M.C. by MIUR PRIN 2005, Prot. 200522492

Correspondence and requests for materials should be addressed to A.J.M.

Supplementary Information accompanies this paper on www.nature.com/naturephysics.

\section{Author contributions}

All authors contributed to the conception and planning of the project, the interpretation of the results and the writing of the paper. The QMC calculations were implemented and carried out by A.C. and the exact diagonalization calculations by M.C. and L.d.M.

Reprints and permission information is available online at http://npg.nature.com/reprintsandpermissions/ 